\title{
Expression, Purification and Binding Activity of Ripening Inhibitor (RIN) protein of Tomato Fruit
}

$$
\begin{aligned}
& \text { Ling } \mathrm{Li}^{1,2, a} \text {, Lu Liang }{ }^{1, b} \text {, Yunxi Liu }{ }^{1, c} \text {, Yanxiu Liu }{ }^{1, d}, \text { Xiaodan } \mathrm{Li}^{1, \mathrm{e}} \text {, and Tieling } \\
& \operatorname{Liu}^{1,2, f, ~ *}
\end{aligned}
$$

${ }^{1}$ College of Food Science and Biotechnology, Tianjin Agricultural University, Tianjin 300384, China

${ }^{2}$ Tianjin Engineering and Technology Research Center of Agricultural Products Processing, Tianjin 300384, China

a lixiaog13@126.com, 'Lianglu1995@126.com, 'Liuyunxi0903@163.com, dLYX610242348@163.com, IIxiaodan@163.com, It|t|@tjau.edu.cn

*To whom correspondence should be addressed.

Key words: Tomato; RIN protein; Prokaryotic expression; Protein purification; EMSA

Abstract. RIN protein plays a very important role in tomato fruit growth, development and ripening process. In order to study the regulation mechanism of RIN transcription factor in tomato fruit, the high purity RIN protein was firstly needed to get. In the study, RIN protein was efficiently expressed through the pET-RIN prokaryotic expression vector, $1.0 \mathrm{mmol} / \mathrm{L}$ IPTG induction $3 \mathrm{~h}$ at $37^{\circ} \mathrm{C}$. The recombinant protein RIN was mainly in inclusion body form $12 \%$ SDS-PAGE analysis. High purity of HIS-RIN fusion protein was gotten by affinity chromatography purification with the Ni Sepharose 6 Fast Flow. After renaturation of RIN protein, the binding activity of RIN protein was identified. The results showed that the renaturation of RIN protein was successfully obtained, and RIN transcription factor has the ability to combine the promoter of LeEXP1 and TBG4 gene. So the protein can be used for further molecular biology research.

\section{Introduction}

Tomato is a typical climacteric fruit, easily softened and perishable, difficult preservation and storage, that's why post-harvest loss accounts for $20 \%-30 \%$ in the total every year. So to ascertain the mechanism of ripe tomato fruit softening has been a hot and difficult research. With the in-depth study of ripening inhibitor (rin), it was found that ethylene and respiration peak disappear, slowly softening in the rin mutant tomato [1,2], so LeRIN gene plays an important role in tomato fruit ripening process. Transcription factor RIN plays an important role in the protein level. Therefore in order to study RIN at the protein level, obtaining the high-purity protein RIN is the primary one.

Affinity chromatography is a useful tool in separation, purification and the analysis of biomacromolecule, especially proteins [3]. In recent years, affinity chromatography is widely used in separation and purification of target protein, and takes a significant part in separation and purification of fusion protein. Electrophoresis mobility shift assay (EMSA) is a simple, fast and extremely sensitive technology in the study of transcription factor protein and binding DNA in vitro [4-7]. This technology has been combined with other technologies and widely used in various fields [8-10]. In eukaryotes, transcription factor, as trans-acting factor, is specifically bound with cis-acting elements in gene promoter, thus plays a inhibition role [11].

At present, domestic research on RIN protein regulation mechanism in tomato fruit ripening is 
seldom. In order to study the regulation mechanism of RIN, we first need to obtain a high-purity RIN protein. In this study, we firstly took the constructed prokaryotic expression vector pET-RIN to express RIN protein. Then, RIN protein had been purification by affinity chromatography, and refolded by urea concentration. The activity of RIN protein was appraised by EMSA finally.

\section{Materials and Methods}

Materials. Recombinant vector pET-RIN, stored in our laboratory strains, was used in the study.

RIN protein prokaryotic expression. Single positive colonies were picked and cultivated overnight in LB liquid medium at $37^{\circ} \mathrm{C}, 200 \mathrm{r} / \mathrm{min}$. In the next day, the substrate was inoculated into fresh LB liquid medium, and cultured 2 to $3 \mathrm{~h}$ until $\mathrm{OD}_{600}$ were up to 0.5 to 0.7 . The final concentration of IPTG was $1.0 \mathrm{mmol} / \mathrm{L}$. $4 \mathrm{~mL}$ cell lysates, which the final concentration of lysozyme was $1 \mathrm{mg} / \mathrm{mL}$, was added in per gram bacterial cell. After that, the bacterial cell was sonicated at $400 \mathrm{~W}$ power, working 10s, interval 10s, total 20 times.

RIN protein affinity chromatography purification. Protein purification method was refered to Ni Sepharose 6 Fast protocol (GE Health).

RIN protein renaturation. RIN was refolded using dialysis solution, containing gradient concentration of urea. The time of samples in each dialysis solution was 8 hours.

transcription factors of gel block experiment (EMSA). EMSA was performed with non-radioactive EMSA kits. The biotin-labeled oligonucleotide probes and unlabeled probes were synthesized from Viagene (USA). The assay was according to the manufacturer's instructions.

\section{Results and Discussion}

RIN protein prokaryotic expression. We picked BL21 single positive colony, containing the recombinant plasmid pET-RIN, in LB liquid culture medium overnight, containing Kan $(100 \mu \mathrm{g} / \mathrm{mL})$. The final concentration of inducer IPTG was $1.0 \mathrm{mmol} / \mathrm{L}$. Induced cells were collected after $3 \mathrm{~h}$ at $37^{\circ} \mathrm{C}$. After sonication, supernatant and precipitate were retained and analyzed with $12 \%$ SDS-PAGE electrophoresis analysis (Fig. 1). It was seen that protein expression was significantly increased in the precipitate of cell lysis solution at $36 \mathrm{kDa}$ (Fig. 1, Lane 3 and 4), and RIN molecular and experimental design fusion protein molecular weight match. It was shown that RIN recombinant protein quickly enriched, aggregated and formed inclusion body.

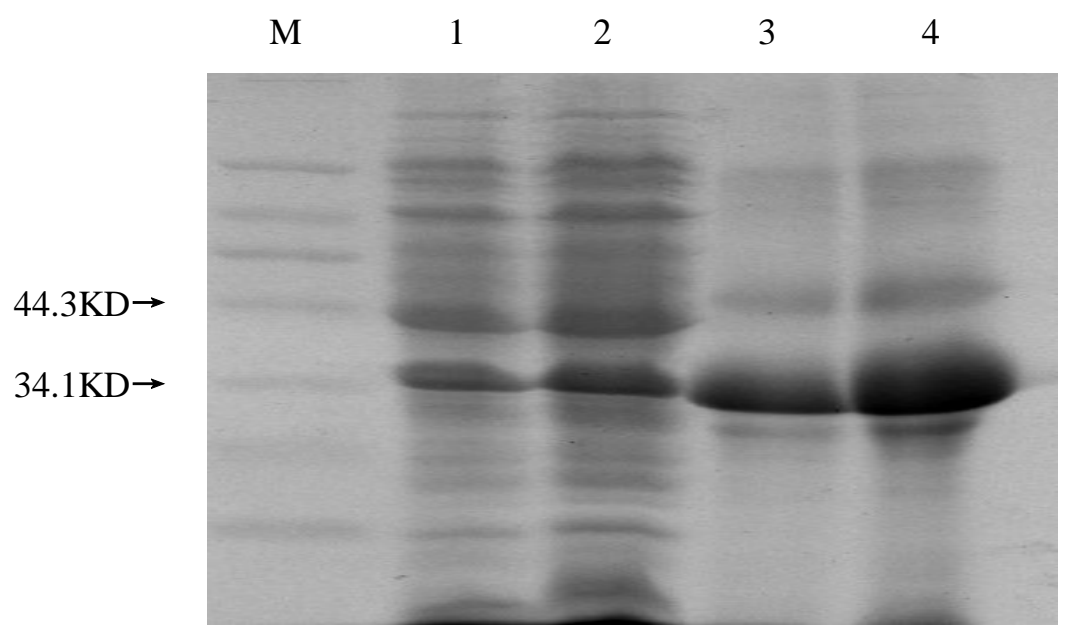

Fig. 1 The prokaryotic expression of RIN recombinant protein

M, Marker; lane 1, 2, supernatant of cell lysis liquid; lane 3, 4, precipitate of cell lysis liquid 
RIN recombinant protein purification. Inclusion bodies were washed according to Leal et al [12], and then resuspended via binding buffer. The supernatant was dissolved with urea and purified in $\mathrm{Ni}$ Sepharose 6 Fast Flow affinity chromatography. RIN recombinant protein was the most abundant protein in the supernatant (Fig. 2, Lane 1). The vast majority of RIN recombinant protein bound to affinity column. A single protein were received in turn according to elution buffer I (Lane 4,5), containing $60 \mathrm{mmol} / \mathrm{L}$ imidazole, elution buffer II (Lane 6,7), including $100 \mathrm{mmol} / \mathrm{L}$ imidazole, elution buffer III (Lane 8,13), embodying $300 \mathrm{mmol} / \mathrm{L}$ imidazole.

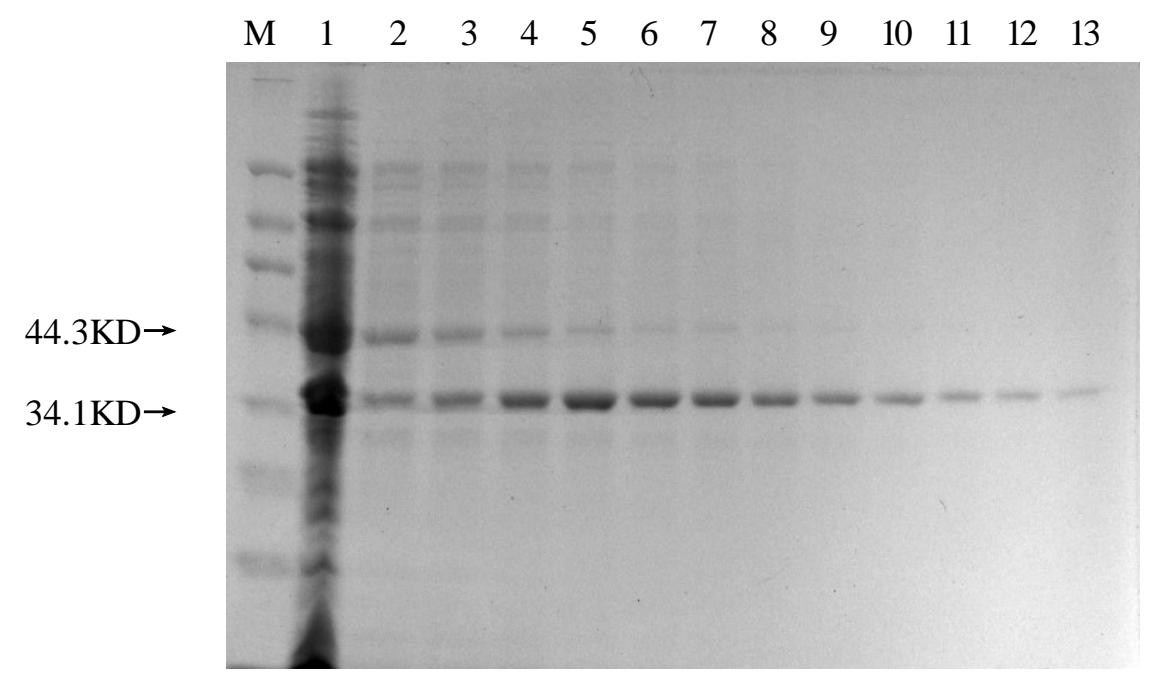

Fig. 2 The purification of RIN recombinant protein. M, Marker; 1, degeneration dissolving of RIN recombinant protein; 2,3, the protein after washing buffer elution

RIN Protein renaturation. In order to obtain biologically active protein RIN, RIN was refolded using dialysis solution, containing gradient concentration of urea. The concentration of urea was followed 6, 4, 2, 1.5, 1, 0.5 and 0 mol/l. As shown in Fig. 3, most supernatant contained soluble and refolded recombinant RIN protein, but precipitate also included small amounts inclusion body RIN.

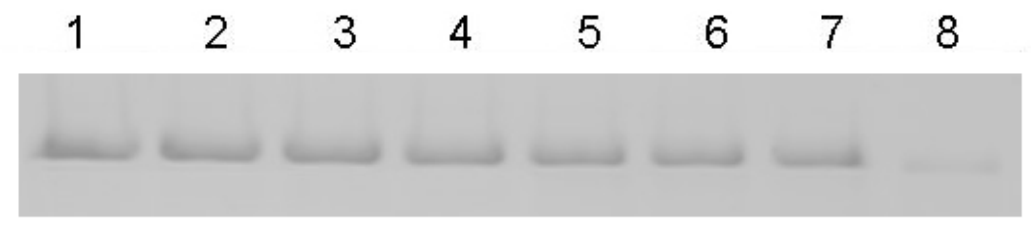

Fig. 3 The solubility of renaturation RIN. 1-7, the supernatant of $6,4,2,1.5,1,0.5$ and $0 \mathrm{~mol} / \mathrm{l}$ urea; 8 , the precipitation after dialysis

The test results of RIN protein gel block in vitro analysis. The electrophoresis mobility shift assay (EMSA) was performed by using biotin-labeled probes containing the CArG motif to evaluate whether or not the RIN protein had the capacity to bind the promoter of LeEXP1 and TBG4 gene in vitro. Purified protein from Escherichia coli cultures harbouring the pET30a vector without the RIN gene was isolated as a control (HIS control). The incubation of HIS-RIN fusion protein with LeEXP1 and TBG4 promoter fragments resulted in DNA-protein complexes that showed an electrophoretic mobility shift, which moved more slowly than the free probe (Lane 3 and 8; Fig. 4B). No DNA-protein complexes formed between the promoter fragments and the HIS control (Lane 2 and 7; Fig. 4B). Competition experiments were conducted to examine the specificity of the mobility shift. The shifted signals for the DNA-protein complexes were weaken and absent when 20 -fold and 100-fold molar excess of the unlabelled competitor was added to the assay respectively 
(Lane 4 and 9, Lane 5 and 10; Fig. 4B). The results demonstrated that the HIS-RIN fusion protein had the capacity of binding to the LeEXPI and TBG4 promoter.
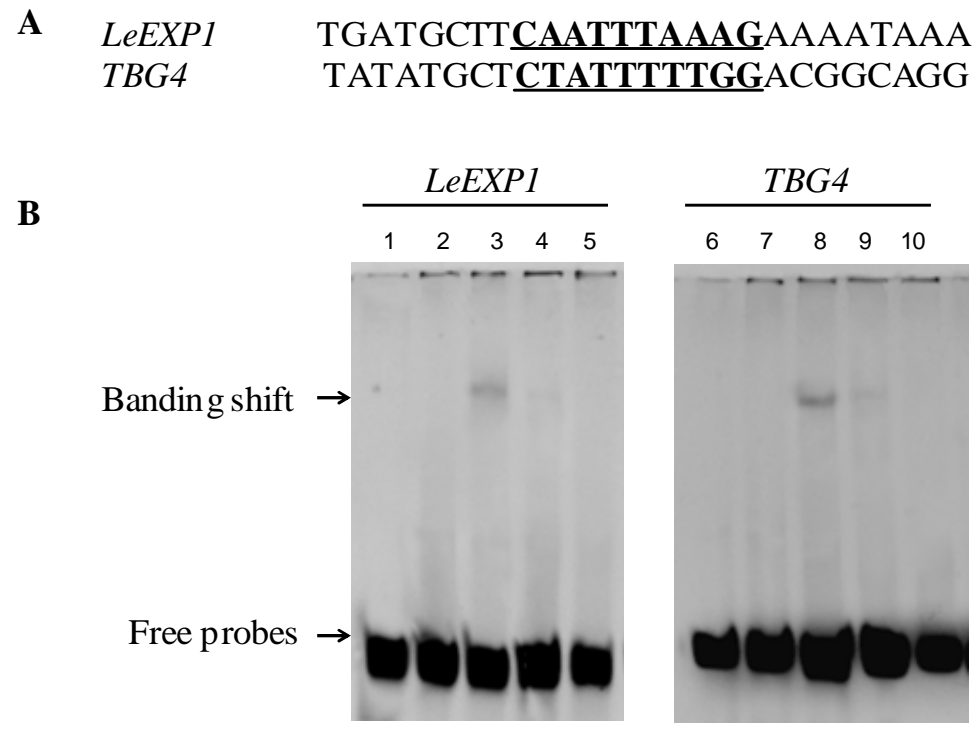

Fig 4 Characterization of the DNA binding activation ability of RIN. (A) Nucleotide sequences of probes. (B) Gel retardation assays with TBG4 and LeEXPl fragments and the recombinant histidine (HIS)-RIN fusion protein. Lane 1 and 6, free probe.

\section{Conclusion}

In the study, RIN protein efficiently expressed, and formed inclusion body. Subsequently, high purity of RIN denatured protein was got. After that, in order to get biological activity of RIN proteins, RIN protein was successfully refolded. At last, it was proved that RIN fusion protein had DNA binding ability in vitro.

\section{Acknowledgements}

This work was supported by grants from the National Nature Science Foundation of China (no. 31201662) and Innovative Training Program of Tianjin College Students (no. 201510061107).

\section{References}

[1] J.Vrebalov, D.Ruezinsky, V.Padmanabhan, et al, Science, 296(2002), p. 343-346.

[2] M.Kitagawa, H.Ito, T.Shiina, et al, Physiologia Plantarum, 123(2005), p. 331-338.

[3] L.Wang, D.J.Liu, L.Z.Li, et al, Physical and Chemical Inspection, 6(2007), p. 515-517.

[4] J.B.Zhang, Z.X.Pan, F.Lin, et al, Hereditas, 31(2009), p. 325-336.

[5] S.Rousseau, J.Renaud, A.Ruiz-Carrillo, Nucleic Acids Research, 17(1989), p. 7495-7511.

[6] M.G.Fried, M.A. Daugherty, Electrophoresis, 19(1998), p. 1247-1253.

[7] P.L.Molloy, Methods in molecular biology (Clifton, N.J.), 130(2000), p. 235-246.

[8] M.Vaculovicova, V.Adam, P.Kizek, Electrophoresis, 36(2015), p. 1084-1085.

[9] Y.Zhang and T.H.Wang, Electrophoresis, 36(2015), p. 1011-1015.

[10] Y.C.Pan, K.Karns, A.E. Herr, Electrophoresis, 35(2014), p. 078-2090.

[11] Y.J.Liu, Northeast Forestry University, (2013)

[12] A.T. Leal, P.C. Pohl, C.A.S. Ferreira, et al. Protein Expression and Purification, 45(2006), p. $107-114$. 